\title{
In vitro and In Silico Studies on Curcumin and Its Analogues as Dual Inhibitors for cyclooxygenase-1 (COX-1) and cyclooxygenase-2 (COX-2)
}

\author{
Nunung Yuniarti ${ }^{1}$, Perdana Adhi Nugroho ${ }^{2}$, Aditya Asyhar ${ }^{2}$, Sardjiman $^{3}$, \\ Zullies Ikawati ${ }^{1} \&$ Enade Perdana Istyastono ${ }^{4}$ \\ ${ }^{1}$ Department of Pharmacology and Clinical Pharmacy, Faculty of Pharmacy, \\ Universitas Gadjah Mada, Yogyakarta, Indonesia \\ ${ }^{2}$ Curcumin Research Centre, Faculty of Pharmacy, Universitas Gadjah Mada, \\ Yogyakarta, Indonesia \\ ${ }^{3}$ Department of Pharmaceutical Chemistry, Faculty of Pharmacy, \\ Universitas Gadjah Mada, Yogyakarta, Indonesia \\ ${ }^{4}$ Leiden/Amsterdam Center for Drug Research (LACDR), Division of Medicinal \\ Chemistry, Department of Pharmacochemistry, Faculty of Exact Sciences, Vrije \\ University Amsterdam, De Boelelaan 1083, 1081 HV Amsterdam, The Netherlands \\ Email: nungki_fa97@yahoo.com
}

\begin{abstract}
Curcumin has been widely reported as an anti-inflammatory agent isolated from the plant Curcuma longa L. (turmeric). This anti-inflammatory activity was associated with the ability of this compound to inhibit the activity of both cyclooxygenase-1 (COX-1) and cyclooxygenase-2 (COX-2) in arachidonic acid metabolism. Dual COX-1 and COX-2 inhibitors are preferred to be employed in the therapy of chronic inflammatory diseases compared to selective inhibitors, since it was reported that the use of selective inhibitors led to severe adverse side effect. In the present study, in vitro and in silico assays on curcumin and its analogues as dual inhibitors for both COX-1 and COX-2 were performed. The results provide theoretical contribution in understanding the ligand-protein interactions at the molecular level to develop new curcumin analogues which possess better anti-inflammatory activity as well as to avoid unsolicited side effects.
\end{abstract}

Keywords: curcumin; cyclooxygenase-1; cyclooxygenase-2; in silico docking; in vitro inhibition assay; dual inhibitor.

\section{$1 \quad$ Introduction}

Curcumin (Figure 1; compound 1a and 1b), the yellow pigment isolated from the plant Curcuma longa L. (turmeric), has been reported to have antiinflammatory activity, as well as antiviral, antioxidant and anti-infectious activities $[1,2]$. Moreover, in several studies, curcumin caused suppression, retardation, or inversion of carcinogenesis [3-6]. The ability of curcumin to inhibit prostaglandins (PGs) biosynthesis in the arachidonic acid cascade has

Received March $5^{\text {th }}, 2010$, Revised November $3^{\text {rd }}, 2011$, Accepted for publication November $21^{\text {st }}, 2011$. Copyright (C) 2012 Published by LPPM ITB, ISSN: 1978-3043, DOI: 10.5614/itbj.sci.2012.44.1.5 
been suggested as an important factor for its anti-inflammatory activity and its anticarcinogenic action [2-7]. Enzyme cyclooxygenases (COXs), which consist of at least two isoforms, i.e., cyclooxygenase-1 (COX-1) and cyclooxygenase-2 (COX-2), catalyze the conversion of arachidonic acid to $\mathrm{PGH}_{2}$ in the first two steps $[3,7,8]$. The first isoform, COX-1, has been suggested as a constitutively expressed enzyme, which is responsible for maintaining normal physiological function $[3,8]$. On the other hand, the second isoform, COX-2 is inducible and the expression is stimulated in the inflammation process [7-9]. At the beginning of the discovery of COX-2, therefore, the selectivity towards COX-2 was considered as beneficial [10]. However, the withdrawal of Rofecoxib, a selective COX-2 inhibitor, due to its increased risk of cardiovascular events, raises an issue that the development of the dual inhibitor of the COX-1 and COX-2 is of considerable interest [8]. Even more, the COX-1 and COX-2 dual inhibitors are suggested to be more appropriate for treating chronic inflammation [11].
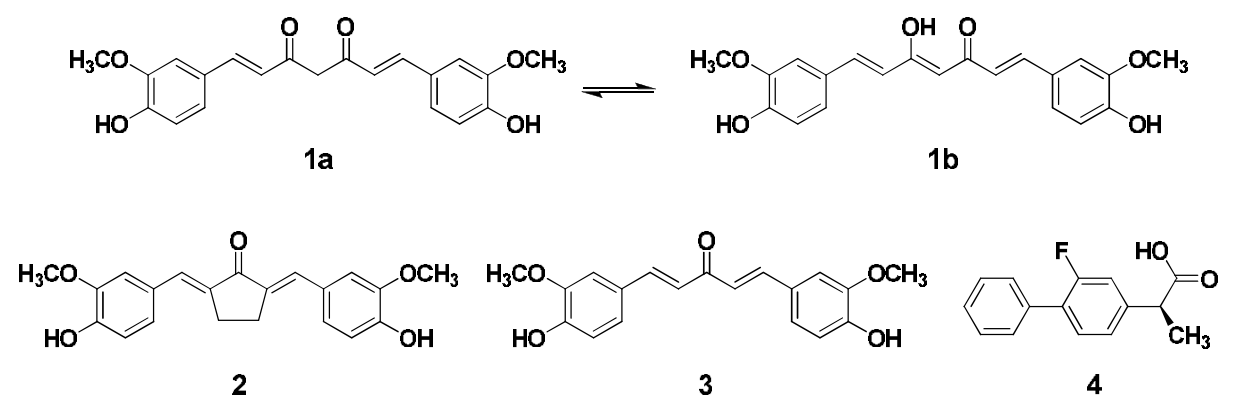

Figure 1 Structures of curcumin (1) in the keto (1a) and the enol (1b) forms, 2,5-bis(4-hydroxy-3-methoxybenzilidene)-cyclopentanone (2), 1,5-bis(4hydroxy-3-methoxyphenyl)penta-1,4-dien-3-one (3), and flurbiprofen (4).

Curcumin inhibits the PGs biosynthesis by inhibiting the activity of cyclooxygenases (COXs), both cyclooxygenase-1 (COX-1) and cyclooxygenase-2 (COX-2) [2-7]. The anti-inflammatory activity of curcumin was related to its ability in inhibiting COX-2 as well as lipoxygenase (LOX) [7, 12]. However, a recent study by Hong et al. [4] suggested that curcumin is more selective to ovine COX-1 compared to ovine COX-2. This result led Handler et al. [3] to synthesize some curcumin analogues as selective COX-1 inhibitors. On the other hand, those results also indicated that curcumin binds to COX-2 as well as to COX-1 and results in a significant inhibition [3,4]. Moreover, Padhye et al. [13] has reported recently that fluorocurcumin is a COX-2 inhibitor. Curcumin, therefore, can serve as a lead compound to develop new inhibitors for both COX-1 and COX-2. 
In silico study in drug design and discovery has flourished together with the advance of computer technology [14,15]. The method is promising to increase the efficiency and effectiveness of the drug and design processes [14]. A number of in silico studies on curcumin have also been reported recently $[3,13$, 16,17]. Handler et al. [3] constructed models by using docking method to explain the activity of curcumin and its analogue as selective COX-1 inhibitors. Other models have been constructed by Padhye et al. [13] to explain the activity of curcumin and its fluoro analogues as selective COX-2 inhibitors. Besides docking method, another in silico approach named quantitative structureactivity relationship (QSAR) has been employed as well [16,17]. Appiah-Opong et al. [16] generated QSAR models of some curcumin analogues as inhibitors of human cytochrome P450 (CYP450) and glutathione S-transferases (GSTs). Moreover, Fujisawa et al. [17] performed a QSAR study on a series of 2methoxyphenols as COX-2 inhibitors with curcumin as one of the studied compound. These studies suggest that in silico study on curcumin and its analogues can perform as a complimentary method in the discovery of new drugs.

This article describes in vitro and in silico studies of some analogues of curcumin (Figure 1), i.e., 2,5-bis(4-hydroxy-3-methoxybenzilidene)cyclopentanone (compound 2), and 1,5-bis(4-hydroxy-3-methoxyphenyl)penta1,4-dien-3-one (compound 3) as well as the parent curcumin molecule, both in keto tautomer (compound 1a) and enol tautomer (compound 1b) as dual inhibitors for COX-1 and COX-2. Compounds $\mathbf{2}$ and $\mathbf{3}$ were selected as the representatives of the curcumin analogues among those in house analogues synthesized by Sardjiman et al. [19] since both have been tested in extensive preclinical studies and have the most similar pharmacophore features with curcumin $[18,19]$.

\section{$2 \quad$ Experimental}

\subsection{Materials}

Curcumin and its analogues were synthesized by Sardjiman et al. [19]. The inhibitory activity of curcumin and its analogues to both COX-1 and COX-2 were measured using Colorimetric COX (ovine) Inhibitor Screening assay purchased from Cayman Chemicals (Cayman Chemical Co., Cat. No. 760111, Ann Arbor, MI, USA).

\subsection{The COX-1 and COX-2 (ovine) Inhibitory Assay}

The assay was carried out using a colorimetric COX (ovine) Inhibitor Screening Assay Kit (Cayman Chemical Co., Cat. No. 760111) as previously described in 
Yang, et al. [20]. The final concentrations of both COX-1 and COX-2 in the assay are $1.45 \% \mathrm{v} / \mathrm{v}(14.5 \mathrm{~mL} / \mathrm{L})$.

\subsection{Molecular Modeling: Hardware and Software}

The docking protocols were performed using Molecular Operating Environment (MOE), version 2008 [10], developed by Chemical Computing Group, Inc. (Montreal, Canada). Interaction fingerprints (IFP) application developed by Marcou and Rognan [21] were performed as the post docking processing. The OpenEye's OEChem1.3 library was used in the IFP computation [21,22]. As long as no further explanation is stated, the default setting was used. All simulations were performed on a Linux (Ubuntu 8.04 LTS Hardy Heron) machine with Intel Core 2 Duo (@2.5 GHz) as the processors and 3.00 GB of RAM.

\subsection{Preparation of Ligand Structures}

Curcumin (both in keto (1a) and enol form (1b)), its analogues (compound 2-4) as well as flurbiprofen (compound $\mathbf{4}$ ) as the reference molecule in the docking simulations were built in the $2 \mathrm{D}$ model in an MOE database viewer. Conformational search was then performed on the database using the Conformational Import module. The results were then washed and minimized.

\subsection{Docking Simulations}

The 3D coordinates of COX-1 and COX-2 were obtained from the protein data bank (http://www.pdb.org/; PDB code: 1eqh and 3pgh, respectively) [23, 24]. Both proteins contain the reference molecule flurbiprofen (compound 4), a dual inhibitor for COX-1 and COX-2 [23]. The binding sites of both proteins were defined by employing the Pocket Selection module in MOE and using the binding sites of flurbiprofen in the crystal structures as the references. Sequences alignments of these binding sites were performed using ClustalW2 [25]. Hydrogens were added using the Protonate 3D module in MOE, and only ligand and protein in chain A were used from the crystal structures. The proteins were subsequently aligned using the Align module in MOE. For each protein, Docking Simulation module in MOE was performed using the database containing prepared ligands as the input file. The results of the docking simulations were subsequently subjected for post docking processing by employing IFP guided visual inspection. The protein-ligand interactions fingerprint of flurbiprofen in the crystal structures were used as the references $[23,24]$. 


\section{$3 \quad$ Result and Discussion}

\subsection{The COX-1 and COX-2 (ovine) Inhibitory Assay}

In the present study, the inhibitory activity of curcumin (1) and its analogues (2 and 3) to COX-1 and COX-2 were tested in vitro and in silico. We found that the compounds are potent inhibitors for both proteins since they could inhibit the activity of the proteins more than $50 \%$ in the concentration of $15 \mu \mathrm{M}$. Some selectivity trends were observed; however the statistical analysis showed that the differences were not significant. At the beginning of the discovery of COX2, which was believed to be more responsible in the inflammatory process compared to COX-1, curcumin was believed to be a more selective COX-2 inhibitor than COX-1 inhibitor [2]. Some previous studies also reported that curcumin is highly related to the COX-2 expression and also binds directly to COX-2 [7]. However, a recent study by Hong et al. [4] suggested that curcumin is more selective to COX-1, which subsequently was supported by Handler et al. [3] by synthesizing some curcumin analogues as selective COX-1 inhibitors. These previous results underlined that curcumin is inhibitor for both COX-1 and COX-2, which was supported by our finding in this study. Moreover, we indicated here that compounds $\mathbf{1 - 3}$ can be potent dual inhibitors for both COX-1 and COX-2. Dual COX-1 and COX-2 inhibitors are preferred to be employed in the therapy of chronic inflammatory diseases compared to selective inhibitors [11], since it was reported that the use of selective inhibitors led to severe adverse side effect $[8,9,11]$.

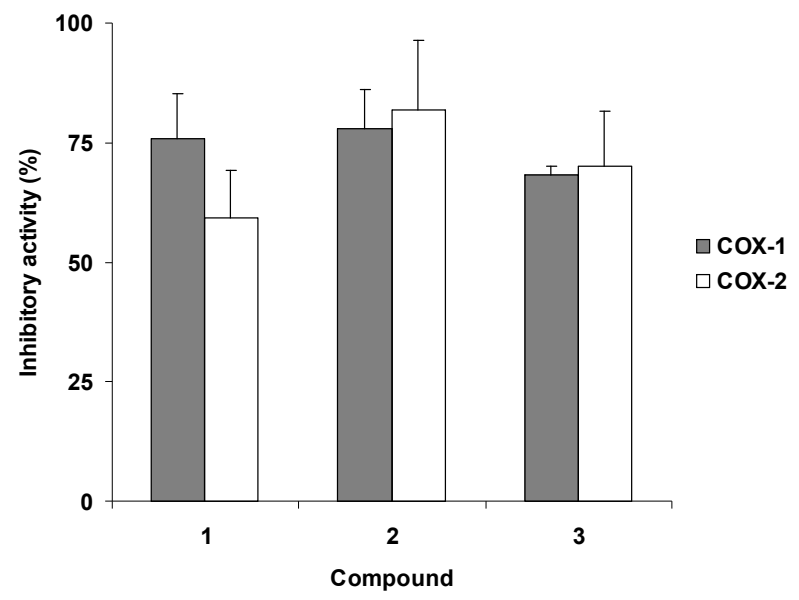

Figure 2 The inhibitory activity of curcumin and its analogues $(15 \mu \mathrm{M})$ to COX-1 and COX-2. The final concentrations of both COX-1 and COX-2 in the assay are $1.45 \% \mathrm{v} / \mathrm{v}(14.5 \mathrm{~mL} / \mathrm{L})$. Data shown are mean $\pm \mathrm{SEM}$ of three independent experiments. 
Figure 2 shows the inhibitory activity of compounds $\mathbf{1}, \mathbf{2}$ and $\mathbf{3}$ to both COX-1 and COX-2. The activities range from $68.4-77.8 \%$ for COX-1 and $59.3-81.9 \%$ for COX-2. Compound 2 shows the highest inhibitory activity for both proteins. Compound $\mathbf{2}$ and $\mathbf{3}$ are more selective towards COX-2, while compound $\mathbf{1}$ is more selective towards COX-1. Statistical analysis using Student's t-test, however, shows that the differences between the inhibitory activities to COX-1 and to COX-2 are not significant at $95 \%$ level of confidence intervals for each compound. Moreover, the ANOVA tests show that the differences between the inhibitory activities of compounds $\mathbf{1}, \mathbf{2}$, and $\mathbf{3}$ are also not significant at $95 \%$ level of confidence intervals for both COX-1 and COX-2.

\subsection{Docking Studies}

The sequences alignments of binding sites using ClustalW2 (Figure 3) have a score of 80 . When only residues that perform direct interaction with the reference compound (flurbiprofen; compound 4) as indicated in IFP analysis were taken into account in the sequence alignments, the score is 94 and 16 out of 17 residues are identical. The residues with residue number 523 (ILE in COX-1 and VAL in COX-2) are found to be the only ones that differentiates the binding site of both enzymes, but still observed as "conserved" in ClustalW2. The cross IFP calculation between the crystal structures gives the Tanimoto metric Interaction Fingerprint (Tc-IFP) of 1.00 for both enzymes. Table 1 presents Tc-IFP of the selected pose for each compound. The Tc-IFPs of the reference compound docking poses are more than 0.6, i.e. 1.00 and 0.95 in the COX-1 and COX-2, respectively. Figure 4 shows the overlay of the crystal structure conformation and the docking pose of compound $\mathbf{4}$ in the binding site of COX-1 (Figure 4A) and COX-2 (Figure 4B). Curcumin (compound 1) in the keto form (1a) possesses a slightly better Tc-IFP value than it is in the enol form (1b) in both COX-1 and COX-2. Figures 5, 6 and 7 present the docking poses of curcumin in the keto form (1a), compound $\mathbf{2}$ and compound $\mathbf{3}$, respectively. Compounds $\mathbf{1}, \mathbf{2}$ and $\mathbf{3}$ are located in the binding site of compound 4 of COX-1 and COX-2. All compounds maintain the interaction with ARG-120 for both proteins (Figure 4-7). Compound 1 maintains all hydrogen bonds possessed by compound 4 in both COX-1 and COX-2 (Figure 5), i.e. to ARG-120 and TYR-355. Compound 1 in COX-1 has one additional hydrogen bond interaction to TYR-385 more than in COX-2 (Figure 5A). Compounds 2 and 3 maintain the hydrogen bond interaction to ARG-120 but lose the interaction to TYR-355. The $\pi-\pi$ interactions of compounds $\mathbf{1 - 3}$ remain similar to compound 4 . 


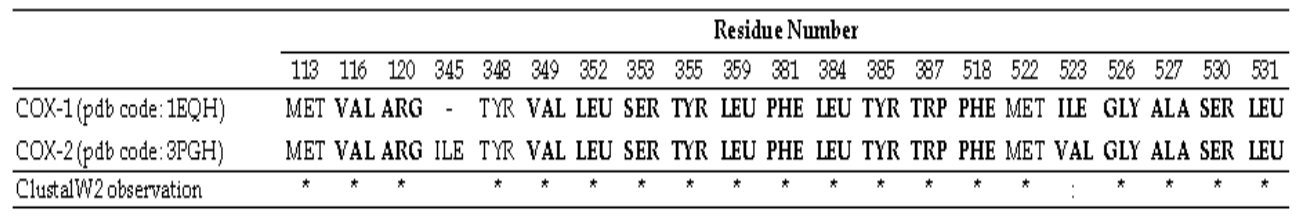

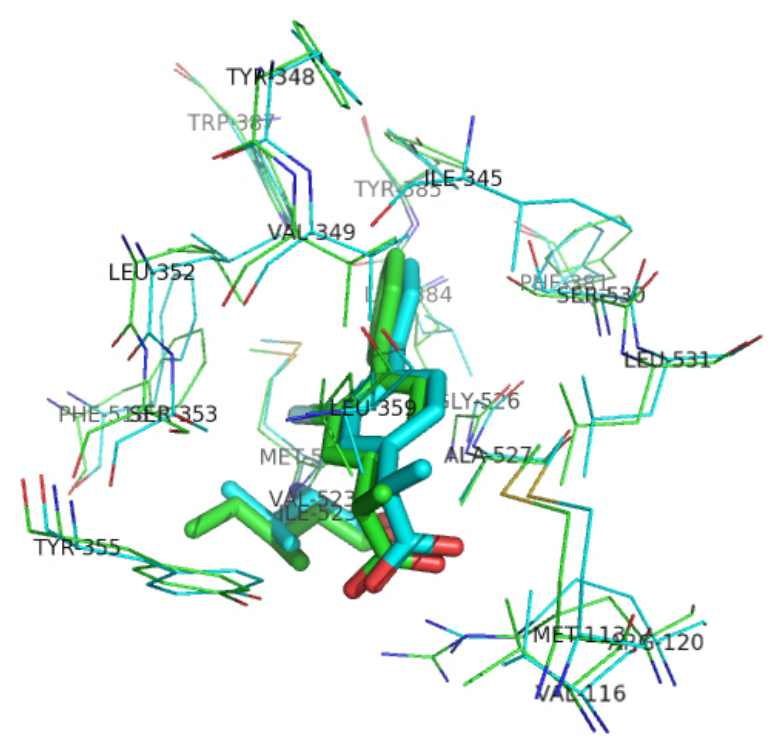

Figure 3 Sequences alignment of the COX-1 (color of carbon atoms are green) and COX-2 (color of carbon atoms are cyan) binding sites. The sequences alignment was performed using ClustalW2, while the figure was generated by employing PyMol v0.99. The alignment shows that the interactions of compound 4 to COX-1 and COX-2 are identical. Compound 4 from both proteins are presented as sticks while the residues in the proteins are presented as lines, except that the residue that differentiates both proteins (ILE-523 in COX-1 and VAL-523 in COX-2) were presented as sticks.

Table 1 The Tanimoto metric Interaction Fingerprint (Tc-IFP) of the selected pose of curcumin (both in keto and enol forms), its analogues and flurbiprofen.

\begin{tabular}{ccc}
\hline \multirow{2}{*}{ Compound } & \multicolumn{2}{c}{ Tc-IFP } \\
\cline { 2 - 3 } & COX-1 & COX-2 \\
\hline $\mathbf{1 a}$ & 0.83 & 0.81 \\
$\mathbf{1 b}$ & 0.78 & 0.80 \\
$\mathbf{2}$ & 0.69 & 0.72 \\
$\mathbf{3}$ & 0.78 & 0.70 \\
$\mathbf{4}$ & 1.00 & 0.95 \\
\hline
\end{tabular}



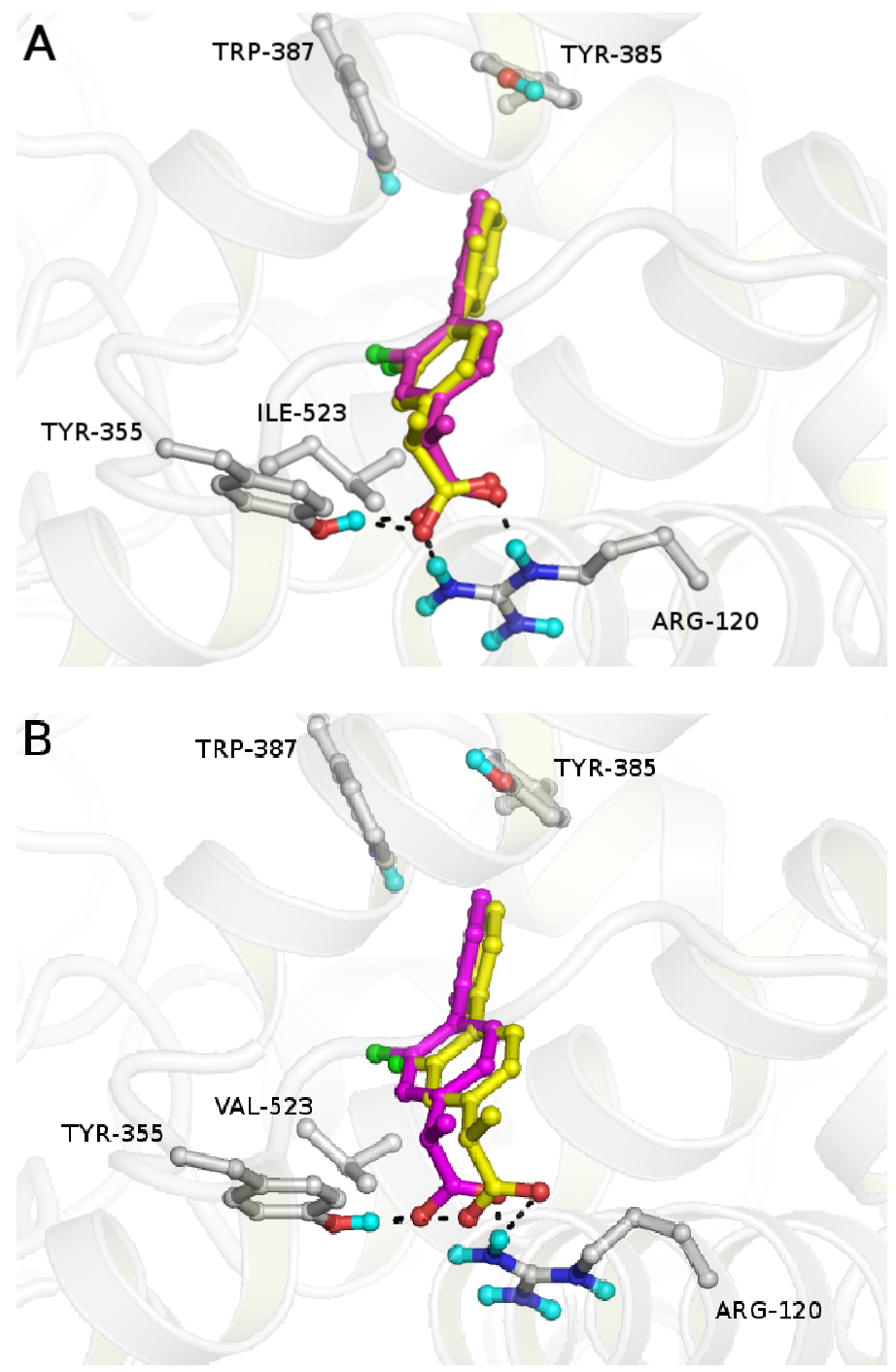

Figure 4 The overlay of the crystal structure conformations (color of carbon atoms are magenta) and the docking poses (color of carbon atoms are yellow) of compound 4 in the binding sites of COX-1 (4A) and COX-2 (4B). For the sake of clarity only residues that have electrostatic interactions $(\pi-\pi$ interaction and hydrogen bonds) with compound $\mathbf{4}$ and the residue that differs from the protein were shown in this figure. Non polar hydrogens are also not shown. Backbone and carbon atoms of the proteins are colored as grey, polar hydrogens are colored as cyan, oxygen atoms are colored as red and fluoro atoms are colored as green. The same depiction settings are applied in Figures 5, 6, and 7. PyMol v0.99 was employed to produce these figures. 


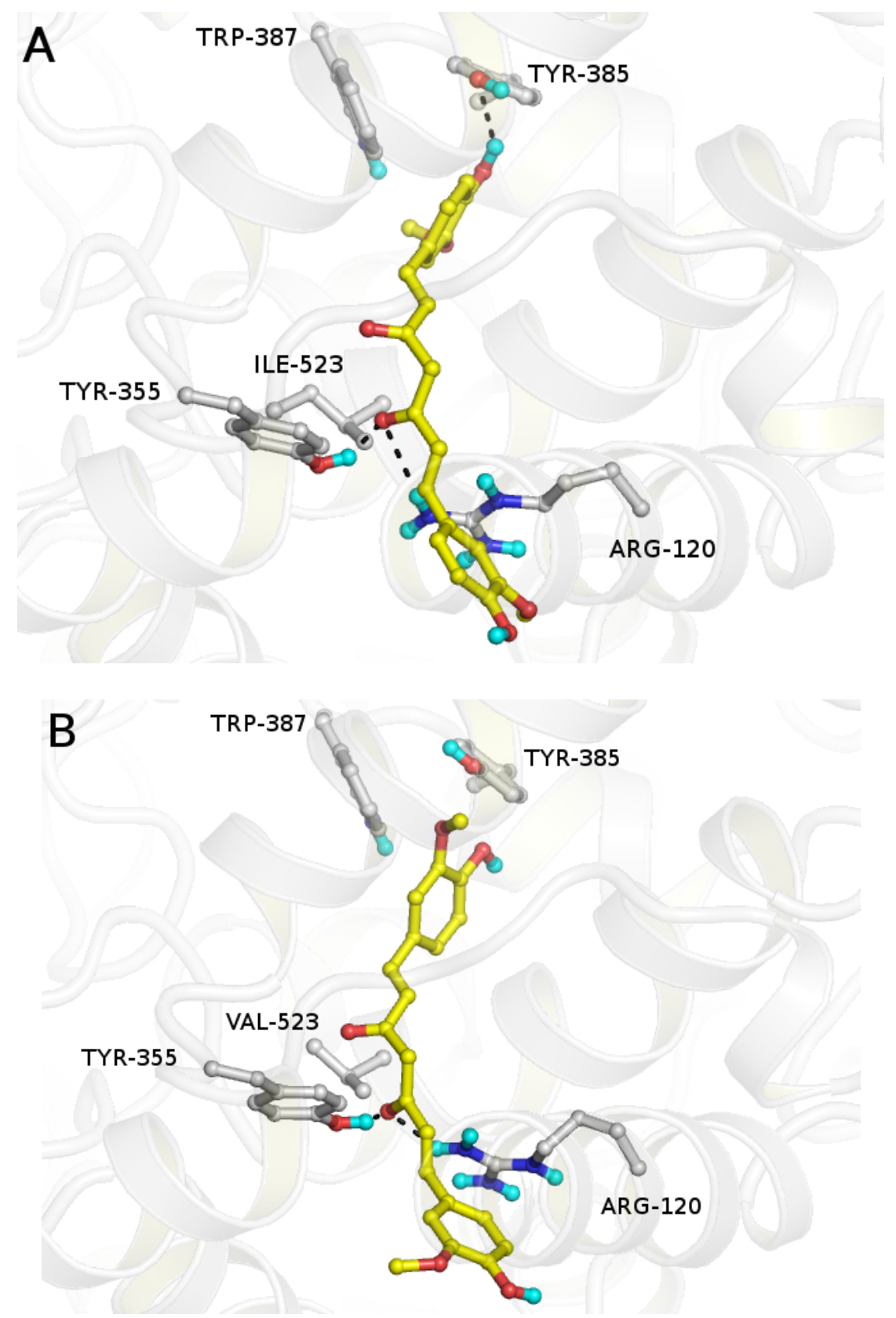

Figure 5 The docking poses (color of carbon atoms are yellow) of compound $\mathbf{1 a}$ in the binding sites of COX-1 (5A) and COX-2 (5B). 

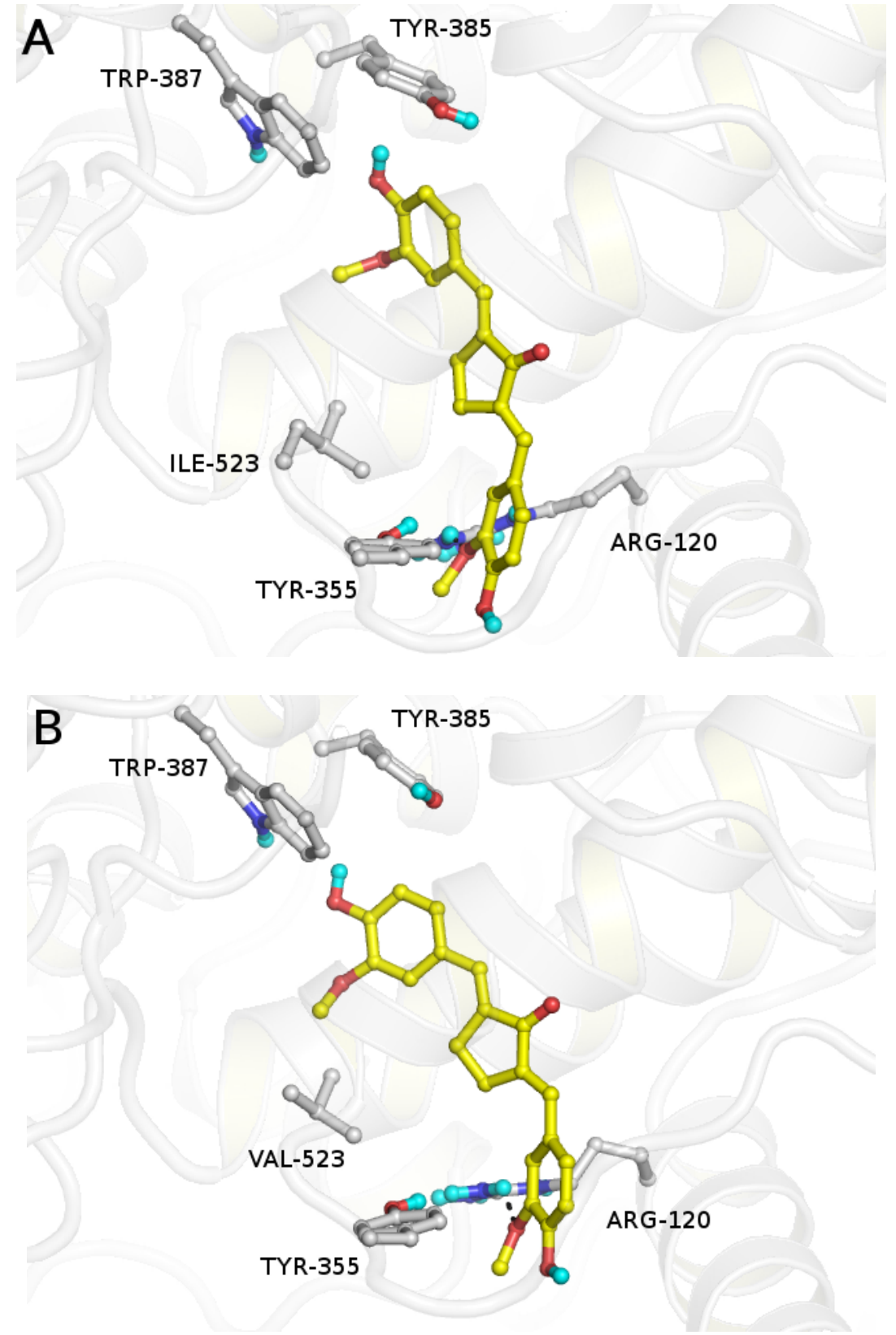

Figure 6 The docking poses (color of carbon atoms are yellow) of compound 2 in the binding sites of COX-1 (6A) and COX-2 (6B). 


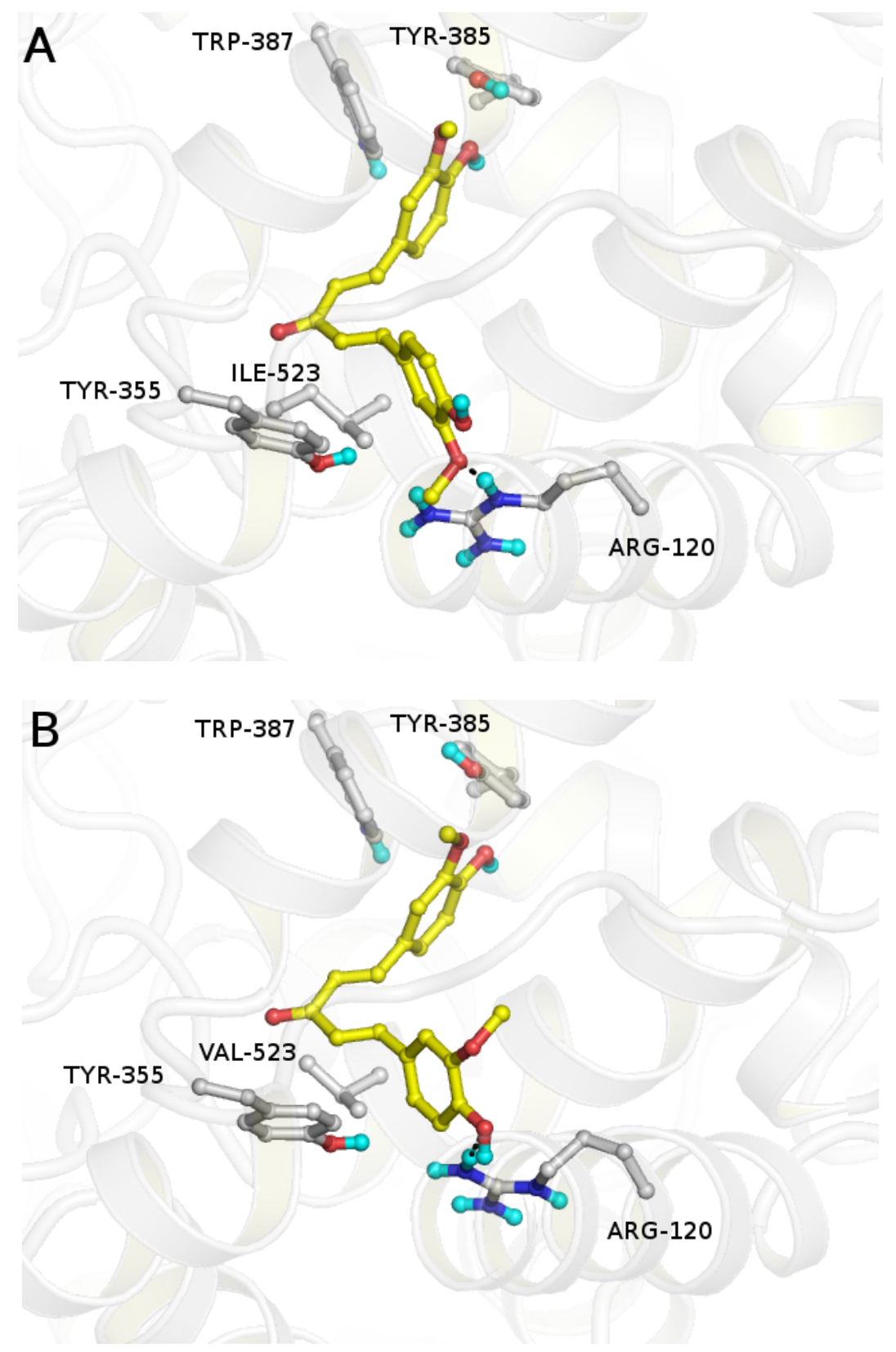

Figure 7 The docking poses (color of carbon atoms are yellow) of compound 3 in the binding sites of COX-1 (7A) and COX-2 (7B). 
In order to support the suggestion that curcumin and its analogues are dual COX-1 and COX-2 inhibitors and to understand the ligand-protein interactions in the molecular level, in silico studies were performed. Crystal structures of COX-1 and COX-2 that contain flurbiprofen (4), a non selective COX-1/COX-2 inhibitor, were used as the models $[23,24]$. The validations of the protocols were performed by re-docking the native substrate, i.e. flurbiprofen $(4)[21,26]$. We found that the docking protocols can reproduce a similar interaction compared to the crystal structure conformations (Tc-IFP $\geq 0.6$ ) $[21,26]$. The in silico screening of compounds $\mathbf{1 - 3}$, therefore, can be performed further using the docking protocols. Compounds 1-3 are fits in the "non-selective" binding sites of COX-1 and COX-2. Compound 1 form hydrogen bonds to residues ARG-120, TYR-355 and TYR-385 in COX-1 but only to residues ARG-120 and TYR-355 in COX-2 (Figure 4). This explains the lower inhibitory activity of compound 1 in COX-2 compared to the inhibitory activity in COX-1. Compound 2 binds in similar manner both in COX-1 and COX-2. It binds to ARG-120. The inhibitory activities of compound 2 in COX-1 and COX-2 are similar. The similar inhibitory activity to both COX-1 and COX-2 is also observed in compound $\mathbf{3}$, which shows similar binding interactions an COX-1 and COX-2. In general, compound $\mathbf{2}$ posses better inhibitory activity compare to compounds $\mathbf{1}$ and $\mathbf{3}$. However, the number of hydrogen bonds formed by compound $\mathbf{2}$ is similar to compound $\mathbf{3}$ and less than compound $\mathbf{1}$. Compared to compounds $\mathbf{1}$ and $\mathbf{3}$, compound $\mathbf{2}$ is more rigid and has less degree of freedom. Thus, compound $\mathbf{2}$ has less entropy compared to compound $\mathbf{1}$ and $\mathbf{3}$. This indicates that the differences of the COX-1 and COX-2 inhibitory activities of compounds 1-3 are determined by the entropy of the system than the enthalpy. These in silico screening results support the in vitro data.

\section{Conclusions}

The present results demonstrate that compounds 1-3 bind in the similar manner with compound 4 in the binding sites of COX-1 and COX-2 which support the in vitro data. Hence, compounds 1-3 could be developed as potent dual COX-1 and COX-2 inhibitors. Moreover, the understanding of the interactions of compounds 1-3 to COX-1 and COX-2 in the molecular level can be further studied in silico. The docking protocols developed in this study can serve also as protocols for further virtual screening of dual COX-1/COX-2 inhibitors.

\section{Acknowledgements}

The authors thank Dr. Chris de Graaf at the Medicinal Chemistry Division, VU University Amsterdam for helpful suggestions in the molecular modeling studies. This work was supported by Directorate of Higher Education, 
Department of Education Government of Indonesian Republic through Hibah Kompetensi 2009 Research Grant.

\section{References}

[1] Joe, B., Vijaykumar, M., \& Lokesh, B.R., Biological Properties of Curcumin-Cellular and Molecular Mechanisms of Action, Crit. Rev. Food Sci. Nutr., 44, pp. 97-111, 2004.

[2] Sharma, R.A., Gescher, A.J., \& Steward, W.P., Curcumin: The Story So Far, Eur. J. Cancer, 41, pp. 1955-1968, 2005.

[3] Handler, N., Jaeger, W., Puschacher, H., Leisser, K., \& Erker, T., Synthesis of Novel Curcumin Analogues and Their Evaluation as Selective Cyclooxygenase-1 (COX-1) Inhibitors, Chem. Pharm. Bull., 55, pp. 64-71, 2007.

[4] Hong, J., Bose, M., Ju, J., Ryu, J.H., Chen, X., Sang, S., Lee, M.J., \& Yang, C.S., Modulation of Arachidonic Acid Metabolism by Curcumin and Related $\beta$-Diketone Derivatives: Effects on Cytosolic Phospholipase $A_{2}$, Cyclooxygenases and 5-Lipoxygenase, Carcinogenesis, 25, pp. 16711679, 2004.

[5] Weber, W.M., Hunsaker, L.A., Roybal, C.N., Bobrovnikova-Marjon, E.V., Abcouwer, S.F., Royer, R.E., Deck, L.M., \& Vander Jagt D. L., Activation of $N F \kappa B$ is Inhibited by Curcumin and Related Enones, Bioorg. Med. Chem., 14, pp. 2450-2461, 2006.

[6] Woo H. B., Shin W. S., Lee S., \& Ahn C. M., Synthesis of Novel Curcumin Mimics With Asymmetrical Units And Their Anti-Angiogenic Activity, Bioorg. Med. Chem. Lett., 15, 3782-3786, 2005.

[7] Zhang F., Altorki N. K., Mestre J. R., Subbaramaiah K., \& Dannenberg A. J., Curcumin Inhibits Cyclooxygenase-2 Transcription in Bile Acidand Phorbol Ester-Treated Human Gastrointestinal Epithelial Cells, Carcinogenesis, 20, pp. 445-451, 1999.

[8] Zambre, A.P., Ganure, A.L., Shinde, D.B., \& Kulkarni, V.M., Perspective Assessment of COX-1 and COX-2 Selectivity of Nonsteroidal 
Anti-Inflammatory Drugs from Clinical Practice: Use of Genetic Function Approximation, J. Chem. Inf. Model., 47, pp. 635-643, 2007.

[9] Dvory-Sobol, H. \& Arber, N., Cyclooxygenase-2 as Target for Chemopreventive Interventions: New Approaches, Cancer Biomarkers, 3 (3), pp. 153-161, 2007.

[10] van Ryn, J. \& Pairet, M., Selective Cyclooxygenase-2 Inhihitors: Pharmacology, Clinical Effects And Therapeutic Potential, Expert Opin. Investig. Drugs, 6, pp. 609-614, 1997.

[11] Gilroy, D.W., Tomlinson, A., \& Willoughby, D.A., Differential Effects of Inhibition of Isoforms of Cyclooxygenase (COX-1, COX-2) in Chronic Inflammation, Inflamm. Res., 47, pp. 79-85, 1998.

[12] Saw, C.L., Huang,Y.I., \& Cong, A.N., Synergistic Anti-Inflammatory Effects of Low Doses of Curcumin in Combination with Polyunsaturated Fatty Acids: Docosahexaenoic Acid or Eicosapentaenoic Acid, Biochem. Pharmacol., 2009.

[13] Padhye, S., Banerjee, S., Chavan, D., Pandye, S., Swamy, K.V., Ali, S., Li, J., Dou, Q.P., \& Sarkar, F.H., Fluorocurcumins as Cyclooxygenase-2 Inhibitor: Molecular Docking, Pharmacokinetics and Tissue Distribution in Mice, Pharm. Res., 26, pp. 2438-2445, 2009.

[14] Kapetanovic, I.M., Computer-Aided Drug Discovery and Development (CADDD): in Silico-Chemico-Biological Approach, Chem. Biol. Interact., 171, pp. 165-176, 2008.

[15] van Gisteren, W.F., Bakowies, D., Baron, R., Chandrasekhar, I., Christen, M., Daura, X., Gee, P., Geerke, D.P., Glattli, A., Hunenberger, P.H., Kastenholz, M.A., Oostenbrink, C., Schenk, M., Trzesniak, D., van der Vegt, N.F., \& Yu, H.B., Biomolecular Modeling: Goals, Problems, Perspectives, Angew. Chem. Int. Ed. Engl., 45, 4064-4092, 2006.

[16] Appiah-Opong, R., Commandeur, J.N., Istyastono, E., Bogaards, J.J., \& Vermeulen, N.P., Inhibition of Human Glutathione S-Transferases by Curcumin and Analogues, Xenobiotica, 39, pp. 302-311, 2009. 
[17] Fujisawa, S., Ishihara, M., Murakami, Y., Atsumi, T., Kadoma, Y. \& Yokoe, I., Predicting the Biological Activities of 2-Methoxyphenol Antioxidants: Effects of Dimers, In Vivo, 21, pp. 181-188, 2007.

[18] Reksohadiprojo, M.S., Timmerman, H., Sardjiman, Margono, S.A., Martono, S., Sugiyanto, Hakim, L., Nurlaila, Hakim, A.R., Puspitasari, I., Nurrochmad, A., Purwantiningsih, Oetari, \& Tedjo, Y., Derivatives of Benzylidene Cyclohexanone, Benzylidene Cyclopentanone, and Benzylidene Acetone, and Therapeutic Uses Thereof, United States Patent Application Publication No.: US2003092772, 2003.

[19] Sardjiman, Reksohadiprodjo, M.S., Hakim, L., van der Goot, H., \& Timmerman, H., 1,5-Diphenyl-1,4-Pentadiene-3-Ones and Cyclic Analogues as Antioxidative Agents. Synthesis and Structure-Activity Relationship, Eur. J. Med. Chem., 32, pp. 625-630, 1997.

[20] Yang, M.H., Yoon, K.D., Chin, Y.W., Park, J.H., \& Kim, J., Phenolic Compounds with Radical Scavenging and Cyclooxygenase-2 (COX-2) Inhibitory Activities from Dioscorea Opposite, Bioorg. Med. Chem., 17, pp. 2689-2694, 2009.

[21] Marcou, G. \& Rognan, D., Optimizing Fragment and Scaffold Docking by Use of Molecular Interaction Fingerprints, J. Chem. Inf. Model., 47, pp. 195-207, 2007.

[22] OpenEye Scientific Software, Inc., Santa Fe, NM, USA, www.eyesopen.com.

[23] Kurumbail, R.G., Stevens, A.M., Giese, J.K., McDonald, J.J., Stegeman, R.A., Pak, J.Y., Gildehaus, D., Miyashiro, J.M., Penning, T.D., Seibert, K., Isakson, P.C., \& Stallings, W.C., Structural Basis for Selective Inhibition Of Cyclooxygenase-2 by Anti-Inflammatory Agents, Nature, 384, pp. 644-648, 1996.

[24] Selinsky, B.S., Gupta, K., Sharkey, C.T., \& Loll, P.J., Structural Analysis of NSAID Binding by Prostaglandin $\mathrm{H}_{2}$ Synthase: Time-Dependent and Time-Independent Inhibitors Elicit Identical Enzyme Conformations, Biochemistry, 40, pp. 5172-5180, 2001. 
[25] Larkin, M.A., Blackshields, G., Brown, N.P., Chenna, R., McGettigan, P.A., McWilliam, H., Valentin, F., Wallace, I.M., Wilm, A., Lopez, R., Thompson, J.D., Gibson, T.J., \& Higgins, D.G., Clustal $W$ and Clustal $X$ version 2.0., Bioinformatics, 23, pp. 2947-2948, 2007.

[26] de Graaf, C. \& Rognan, D., Selective Structure-Based Virtual Screening for Full and Partial Agonists of the $\beta 2$ Adrenergic Receptor, J. Med. Chem., 51(16), pp. 4978-4985, 2008. 